\title{
La escuela y la cuestión del mérito: reflexiones desde la experiencia chilena
}

Kathya Araujo'

Danilo Martuccelli"

\section{Resumen}

Este artículo discute la importancia y centralidad que ha adquirido el mérito en la sociedad chilena hoy, enfocándose principalmente en sus efectos asociados con la experiencia educacional. El argumento es desarrollado en tres pasos. Luego de una presentación del contexto histórico y de las transformaciones sociales que dan cuenta de su expansión, se analizan sobre todo las maneras en que el reconocimiento del mérito es distintamente evaluado en las diferentes etapas de la trayectoria escolar por las clases medias y los sectores populares. En un tercer momento, se muestra de qué manera a través del extendido recurso a las redes sociales y a los contactos interpersonales, la expansión del mérito se acompaña del surgimiento de un nuevo horizonte de injusticia social. El artículo pone en evidencia, así, a la vez el fuerte consenso en torno al valor del mérito en Chile y los sentimientos de injusticia y frustración que ello engendra en los individuos. La discusión presentada se basa en los resultados de dos estudios cualitativos. La primera investigación se desarrolló en las ciudades de Santiago, Concepción y Valparaíso, entre 2007 y 2009, a través de alrededor de un centenar de entrevistas a hombres y mujeres de entre 30 y 55 años, pertenecientes la mitad de ellos a sectores populares urbanos y la otra mitad a las clases medias y medias-altas. La segunda investigación, en curso, aportó al análisis dieciséis entrevistas hechas en Santiago, a lo largo del 2014, a partir de las cuales se profundizó la problemática del mérito.

\section{Palabras clave}

I- Universidad Academia de Humanismo Cristiano, Santiago, Chile.

Mérito - Redes - Justicia - Sectores populares - Clase media.

Contacto: karaujo@academia.cl

II- Université Paris Descartes, Paris, Francia.

Contacto: danilo.martuccelli@parisdescartes.fr 


\section{School and merit: reflections from the Chilean experience}

Kathya Araujo'

Danilo Martuccelli"

\section{Abstract}

This article discusses the importance and centrality that merit has in Chilean society today, focusing on its multidirectional relationships to the educational realm. Our argument will be developed in three steps. Firstly, we will present the historical context of the social transformations that are to be related with the expansion of the principle of merit in this society. Secondly, we will analyze the manners in which the recognition of merit is evaluated at each of the different stages of school trajectories by members of middle and popular sectors. Finally, we will describe and discuss the way in which, due to the maintained impact of social networks and interpersonal contacts, the expansion of merit is closely related to the emergence of a new horizon of social injustice. Consequently, the article evidences that, concerning the expansion of the principle of merit in Chile, we face a twofold process: the emergence of a strong consensus about the value of merit, and the widespread sentiments of injustice and frustration that it produces in individuals. This discussion is based upon the findings of two qualitative studies. The first one was developed between 2007 and 2009 in Santiago, Concepción and Valparaiso cities, in Chile. About one hundred interviews were held with men and women aged 30 to 55 years of middle and popular sectors. The second, an ongoing study, provided our analysis with sixteen interviews, which were carried out in Santiago in 2014, and enabled us to further analyze the merit theme.

\section{Keywords}

Universidad Academia Humanismo Cristiano, Santiago, Chile.

Merit - Networks - Justice - Popular sectors - Middle classes

Contact: karaujo@academia.cl

Université Paris Descartes, Paris, France.

Contact: danilo.martuccelli@parisdescartes.fr 
En este artículo nos interesaremos por las razones estructurales y las expresiones experienciales por las que el mérito se ha convertido en un horizonte de justicia particularmente activo en la sociedad chilena, y los desafíos que ello supone. El sistema educacional chileno es hoy, en lo que a la justicia se refiere, una combinación entre la permanencia de la segregación de públicos (según los origenes sociales) y una selectividad fuertemente asociada, en sus grandes principios, al mérito personal. Ciertamente, el origen social familiar y el diploma de los padres sigue siendo estadísticamente determinante, como en tantos otros países, a nivel de las trayectorias escolares (DÁVILA; GHIARDO; MEDRANO, 2005; LEÓN; MARTÍNEZ, 2007; RIVAS, 2008) y del tipo de establecimiento educativo al que se concurre público, municipal o privado (MANZI, 2006; BENGOA, 2009). Sin embargo, esta situación no impide en absoluto que la escuela se haya convertido en un factor central del proyecto de movilidad social y de justicia de los chilenos. Pero, si el valor que se otorga a la educación es similar entre las distintas capas sociales, su instrumentalización es, como lo veremos, muy diferente.

Para mostrarlo, nos apoyaremos sobre dos investigaciones empíricas. La primera fue realizada en Chile (en las ciudades de Santiago, Concepción y Valparaíso), entre 2007 y 2009, a través de un centenar de entrevistas semi-directivas a hombres (un total de 48) y mujeres (también un total de 48) de entre 30 y 55 años, pertenecientes la mitad de ellos a sectores populares urbanos y por la otra mitad a las clases medias y medias-altas, cuyo objetivo central fue estudiar las grandes características del proceso de individuación en la sociedad chilena y, dentro de él, los aspectos propiamente escolares (ARAUJO; MARTUCCELLI, 2012) ${ }^{1}$. La segunda, en curso, sobre la constitución

1- Proyecto Fondecyt 1085006 - Procesos de individuación y configuración de sujeto en la sociedad chilena actual. La muestra no consideró definiciones profesionales específicas, pero se cuidó de mantener una cierta representatividad evitando la sobresaturación de ciertos perfiles profesionales. En cambio la pertenencia a sector socioeconómico fue central. Por razones de economía expositiva hemos optado en este artículo por simplificar la posición de las personas entrevistadas en clases medias (que pertenecen a lo que en Chile se denomina el sector $\mathrm{ABC1}$ y C2) y sectores populares (individuos que pertenecen esencialmente al llamado sector $\mathrm{D}$, pero también al $\mathrm{C} 30 \mathrm{E}$ ). de los sujetos en Chile, aportó con el análisis de dieciséis entrevistas, efectuadas en Santiago a lo largo del 2014, en las cuales se profundizó la problemática del mérito ${ }^{2}$. Apoyándonos e ilustrando nuestro razonamiento a partir de estas entrevistas, desarrollaremos la argumentación en tres etapas. En un primer momento, recordaremos rápidamente la importancia del tema del mérito y el contexto histórico en el que éste se ha afırmado como un valor central en la sociedad chilena. En segundo lugar, declinaremos la manera en que los diferentes grupos sociales viven la lógica del mérito en cada uno de los segmentos del sistema educativo. Por último, insistiremos en la fuerza diferencial de las redes y su impacto a la hora de definir un nuevo horizonte de injusticia social.

\section{El mérito como principio de justicia}

La recompensa del mérito es un asunto político no solamente muy sensible en la sociedad chilena contemporánea, sino que incluso es un tema nuevo (ENGEL; NAVIA, 2006; CONTARDO, 2008). Si el proceso es visible en muchos otros países latinoamericanos, la expansión del mérito como valor se asocia en este país con los cambios ocurridos en las últimas décadas y la intensidad con la que se instaló la lógica del mercado. Al mismo tiempo, ella es reflejo de una tendencia mayor del individualismo contemporáneo: aquella que valoriza el esfuerzo y el trabajo y, en particular, su recompensa, ya sea monetaria, ya sea en términos de movilidad social (MICHAUD, 2009; DUBET, 2006). El mérito sintetiza pues un doble ideario: por un lado, se ubica en la estela de una demanda fuertemente legitimada por el modelo neoliberal de éxito personal y, por el otro, y al mismo tiempo, es una prolongación bajo otras coordenadas de un anhelo de igualdad social.

La ideología meritocrática convive en todos lados con un orden social en el cual las herencias falsean permanentemente la justicia

2- Entrevistas que forman parte de los primeros resultados del Proyecto Fondecyt 1140055 - El trabajo de los individuos y la constitución de sujetos en la sociedad chilena. 
enunciada. No es esto, por supuesto, lo que es específico o novedoso en el caso chileno. La novedad reside en la tensión que los individuos perciben y denuncian entre dos tendencias contrarias. Por un lado, la conciencia que jamás el país ha sido tan competitivo y con una fuerte invocación al mérito individual $y$, por el otro lado, la convicción que el reconocimiento del mérito está muy lejos de ser una realidad. Se está, pues, frente a una tensión específica entre los principios de igualdad y de movilidad, en la que la segunda prima sobre la primera a medida que el ideal de la meritocracia se impone (MÉNDEZ; GAYO, 2007). De hecho, como lo veremos, si entre las personas entrevistadas la distorsión del mérito por las lógicas sociales fue muchas veces denunciada, nadie empero criticó el carácter justo del mérito.

Imposible desconocer la importancia del cambio. Sin jamás haber estado completamente ausente, el mérito nunca fue un pilar de la justicia en América Latina. El imaginario de la riqueza no fue sino en muy escasas ocasiones percibido como fruto del mérito individual. El peso de las herencias y los privilegios, pero también de las barreras sociales, hacía poco menos que ilusoria una reivindicación de este tipo. Entre la alcurnia extraída de la cuna, tan bien representada en Chile por los apellidos (STABILI, 2003) y el golpe fortuito de la suerte que sólo les toca a algunos, no había, en verdad, espacio para el mérito. Hoy, por el contrario, el mérito se ha convertido en una fuente indiscutible de valoración personal y evaluación crítica de la sociedad.

Si la expansión actual del mérito testimonia una profundización del individualismo, $\mathrm{y}$ en este sentido no puede ser enteramente disociado de ciertos valores introducidos por el modelo neoliberal, tampoco puede empero ser reducido a él. Ciertamente, la influencia del modelo neoliberal es patente en la valorización de la ambición personal, de la confianza en el esfuerzo propio, de la importancia del empuje, de las ganas de tener éxito. En estos discursos normativos es posible observar, a nivel individual, la expresión de un modelo político colectivo. Los individuos, y esto vale en particular para los sectores de clase media, hablan del mérito como portavoces del sistema. Las frases fueron inequívocas: el que quiere, puede. Sin embargo, a esta influencia es preciso añadirle una fuente adicional. Una en la cual el mérito se asocia preferentemente con la igualdad y la justicia. Una perspectiva que, sin ser exclusiva, está bastante más extendida entre los sectores populares. Sobre todo, y es lo que es imperioso retener, si la exigencia del mérito no se reduce solo al ámbito escolar (puesto que también está muy presente en el mundo del trabajo), su horizonte de justicia es particularmente activa a nivel escolar.

\section{El mérito desde la experiencia escolar: niveles de estudio y miradas cruzadas}

El valor que los chilenos le otorgan a la educación no esperó las últimas décadas para manifestarse, sin embargo, el sentido que se le otorga a la educación ha variado, puesto que cada vez más se la evalúa en función de una promesa individual de movilidad social. Este anhelo es tanto más fuerte que se da en el marco de una sociedad que ha vivido un innegable y fuerte proceso de movilidad educativa (BRUNNER, 2007; GAYO, 2007), que se ha traducido por un aumento sostenido de expectativas escolares al punto que hasta un 70,3 \% de alumnos piensa llegar a completar una formación de nivel universitario (CIDE, 2012). Notémoslo: las generaciones entrevistadas vivieron la fuerte expansión de la matrícula escolar tanto a nivel de la educación básica (que en el 2013 llegó a una cobertura del 95\%), la educación media (con un porcentaje de 92\%) (CHILE, 2013a) e incluso en la enseñanza superior que pasó de 15,6\% en 1990 a 45,8\% en el 2011 (CHILE, 2013b).

Sin embargo, sería un error suponer una generalización uniforme de las expectativas ${ }^{3}$. Más

3- En los apartados siguientes se presentan una serie de fragmentos de las entrevistas realizadas para este estudio con el fin de ilustrar nuestros argumentos. 
allá de la expansión de la cobertura educativa, el mérito se dota de significaciones distintas según los grupos sociales y niveles de estudios, algo que está bien reflejado, en un primer momento, a nivel de los diferenciales de recuerdos que unos y otros poseen a propósito de sus años escolares. También lo hace en el modo en el que la experiencia educativa participa en las maneras en que se constituyen como sujetos, pero, sobre todo, se refleja muy claramente en el hecho de que a medida que se avanza en los estudios se transforma el equilibrio entre una lógica propiamente estratégica y una lógica educativa.

\section{La escuela primaria}

El recuerdo y el relato que los individuos dieron de su experiencia escolar, y de sus consecuencias, fueron muy diferentes en función de los grupos sociales. Es así como, por ejemplo, la escuela básica fue solo y exclusivamente evocada espontáneamente por los hombres y mujeres de sectores populares, en mucho para narrar a la vez las dificultades y los encantos de la infancia, la pobreza, en algunos el maltrato de los profesores, y en otros, la presencia -y la nostalgia- de una escuela con clara función educativa. Sobre todo las mujeres, la evocaron para afirmar hasta qué punto la escuela permitía escapar a las tareas domésticas impuestas por los padres, e incluso, retrospectivamente, creían poder percibir, ahí, y en ese momento, el punto mayor de la bifurcación social: "me hubiera gustado estudiar, pero no se pudo", porque tuvo que entrar a trabajar a los doce años, nos dijo una mujer de sectores populares. Una experiencia tanto más dura que, como es el caso de esta otra mujer, se dio en el marco de lo que vivió como una injusticia familiar, aquella en la cual se escogía a aquel que debía ponerse a trabajar, porque "no podían pagarme otra cosa", pero "igual uno tiene ganas de seguir estudiando" algo que, por el contrario, pudieron hacer otras hermanas..." Los recuerdos son similares en un hombre que solo llegó hasta el fin de la primaria, y que recuerda, lamentándolo, que cuando "le pedía a mi papá que me comprara un cuaderno, me decía "cómo te voy a comprar un cuaderno, no tenemos ni para comer y quieres que te compre un cuaderno..."”

La evocación de los años en la escuela básica está también ligada al recuerdo, más o menos mítico, de haber sido un "súper buen alumno" y que, por ende, el abandono de los estudios, se explica en mucho, retrospectivamente, por la necesidad de entrar a trabajar. Un obrero lo expresó de manera explícita:

[...] no seguí estudiando (por razones económicas), y hasta el día de hoy me arrepiento de no haber seguido estudiando más, porque me quedé con eso (estudios primarios) y eso me significó después no tener otras oportunidades, porque cuando uno pierde el hilo del estudio después cuesta retomarlo.

La escuela, en el recuerdo retrospectivo, reenvía a un horizonte que, contra tantas evidencias, se piensa estaba abierto.

En claro contraste con estas afirmaciones, la ausencia radical de toda referencia a la escuela básica entre los recuerdos significativos de los individuos entrevistados de clases medias tiene un sentido evidente. La carrera educativa no sólo es más larga para ellos, sino que comienza, y se juega de todas formas, más tarde. Es solamente en el secundario y en la universidad, por ende, que la escuela interviene en los relatos, a pesar de que, como veremos, las relaciones que establecen en la escuela los surte de un conjunto de recursos que movilizarán activamente.

\section{La escuela secundaria}

En su conjunto, esta etapa de la escolaridad fue más evocada por los hombres que por las mujeres, tanto en los sectores populares como en las capas medias y altas. El tono no fue empero el mismo. En los testimonios de los sectores populares, la escuela secundaria 
es todavía una época idílica ("lo pasé muy bien - se ríe -, lo pasé muy bien. Un año me lo repetí por la pura cimarra, o sea me divertí todo el año, todo el año..."), y en algunos, incluso, todavía un momento de rememoración de performances escolares, pero ya menos mágico que la escuela básica. En verdad, la escuela secundaria aparece, por lo general, como un momento de inflexión de la carrera escolar: las notas descienden, el universo juvenil se afirma ya no solamente de manera paralela, sino incluso de manera opuesta a la escuela. "Hacía la fiesta", se recuerda este empleado de supermercado, "para ir a jugar a los flippers". Poco importan las razones, para la mayoría de las personas entrevistadas la trayectoria escolar se cortó por la necesidad de insertarse en el mercado de trabajo y por el anhelo de ganarse sus vidas porque "me interesaba la plata de repente".

Para las clases medias, por el contrario, el secundario aparece, en los recuerdos retrospectivos, como un momento de eclosión de una conciencia, sino siempre crítica, por lo menos más personal. Y en todos, sobre todo, cualquiera que haya sido la vivencia efectiva, la evidencia de clase que de todas formas los estudios más o menos largos se inscribían en el horizonte natural de sus trayectorias personales. Pero si el secundario aparece como una etapa en una trayectoria escolar más larga, esto no debe hacer descuidar que, como lo analizaremos en detalle en un parágrafo ulterior, la escuela secundaria tiene en este sector social una función determinante en términos de constitución de su capital social.

En este nivel de estudios, subrayemos igualmente un elemento importante presente, masiva y únicamente, entre los miembros de los sectores populares. A saber: la experiencia del fracaso escolar y, sobre todo, la evidencia de la necesidad de abandonar, frente a las primeras dificultades, los estudios (ya sea por razones económicas o por razones de nivel académico). Los testimonios recabados en torno al fracaso escolar son tanto más importantes que las experiencias de los más jóvenes de nuestra muestra coinciden con la expansión de la matrícula en los colegios particular-subvencionados (establecimientos privados con subvención pública) que pasa del 33\% en 1990 al 51\% en el 2009 (CEPPE, 2011), indicando una toma de conciencia de la importancia estratégica de los estudios entre los sectores populares ${ }^{4}$ y sobre todo; la tendencia a disociar la movilidad social ascendente del sistema educativo público (RACZYNSKI, 2010). Una actitud que contrastó fuertemente con los relatos de los miembros de las capas medias, en donde el devaneo en la orientación y el fracaso escolar fueron posibilidades consentidas por el entorno familiar.

En el corazón del pacto familiar entre generaciones en Chile hoy, se encuentra la idea entre los padres de clases medias, cuyos recursos lo permiten, que deben costear los estudios de sus hijos. Cueste lo que cueste. Un acuerdo tácito que permite tanto alargar las carreras, como equivocarse en la vocación -algo particularmente visible a nivel universitario. La chance nunca se juega en un solo naipe. Es el caso de este ingeniero comercial, quien, por ejemplo, sacó su carrera en seis y no en cinco años, "pero tampoco fue un tema... Por suerte no tenía necesidades económicas imperiosas que me hicieran terminar la carrera rápido, así que, fue muy agradable la universidad". En otros, la segunda chance es una verdad concreta. Luego de estudiar y titularse en biología, una mujer descubrió su "alma artística” y se puso estudiar, siempre con la ayuda de sus padres, una segunda carrera -arquitectura. La experiencia sin ser frecuente no es rara. "La verdad es que tenía mucha desorientación. Yo entré a la Universidad sin saber lo que iba a estudiar". Buena alumna, le fue muy bien en la prueba de aptitud, "así que entré a estudiar odontología sin saber lo que era ser odontólogo". Cuando lo descubre, se cambia de carrera y cursa pedagogía.

4-En el mismo periodo el porcentaje de alumnos inscritos en los colegios municipalizados (públicos) desciende del 59\% en 1990 al 42\% en el 2009. 0 sea, que nuestros entrevistados forman parte de la cohorte en la que se afirmó definitivamente un modelo de enseñanza dominante privada en Chile. 
Imposible desconocer el rol que le toca a este suplemento de tiempo en el proceso de reconocimiento y certificación escolar del mérito. Ahí donde los miembros de los sectores populares no tienen derecho al fracaso, los de las clases medias y altas disfrutan de un suplemento de oportunidad y de tiempo. Para muchos individuos de las clases medias, el descubrimiento de una pasión personal, es un indudable privilegio de clase.

\section{La universidad y los estudios terciarios superiores}

En Chile, los años de universidad aparecen en muchos testimonios a la vez como una combinación de estrategia social y de crecimiento personal. En algunos, la experiencia de descubrimiento de sí mismos, coincide con lo que perciben como un descubrimiento del país, del verdadero país. Si el proceso tiende hoy a decrecer, para varias generaciones de jóvenes de capas medias, la universidad pública fue un viaje en las clases sociales. En la Universidad, como tantos relatos lo evocan, descubrieron el otro Chile. Por primera vez en sus vidas, se encontraban en relación con miembros de otros grupos sociales. Este abogado recuerda cómo,

[...] cuando empecé a estudiar, empecé a notar otras cosas, empecé a notar ciertas diferencias culturales y en eso la Universidad (pública), la facultad, donde yo estudié, era muy rica, en el sentido de que teníamos una diversidad de compañeros de distintas procedencias.

Un médico dio un relato similar: "Yo siempre digo que la Universidad de Chile a mí me salvó porque me sacó de la burbuja. Mi vida en la Universidad me marcó en mi vocación".

Através de los relatos sobre las trayectorias escolares, se va diseñando una importante diferenciación social, a medida que la dimensión propiamente educativa de la enseñanza escolar y universitaria se diferencia entre los grupos sociales. El tema ha sido relativamente descuidado por la sociología de la educación, sobre-orientada hacia los problemas de desigualdad o de calidad del sistema educativo (BRUNNER, 2006). Sin embargo, el punto es importante porque el rol del sistema educativo en la formación del carácter no es el mismo en todas las categorías sociales. En los sectores populares, esta función se reduce al recuerdo de ciertas reglas de vida y de disciplina; en las capas medias, por el contrario, cada nivel de estudio abre -en medio de claros objetivos estratégicos- a una experiencia educativa distinta. Esta diferencia decrece en el país en los últimos años a medida que se incrementa el número de jóvenes de sectores populares que van a la universidad. Pero, así y todo, la diversidad de las trayectorias refleja privilegios sociales innegables que, incluso en términos de formación de la personalidad, cuestionan el rol que la escuela tiene - o debe tener - en la promoción igualitaria del mérito.

Sin embargo, la importancia de este diferencial a nivel de las experiencias educativas propiamente dichas, no debe llevar a descuidar la dimensión propiamente estratégica de los estudios universitarios. Y ello tanto más que, si por el momento globalmente en el país los años suplementarios de estudio se traducen en mejores ingresos (SAPELLI, 2009; MIZALA; ROMAGUERA, 2004; BRAVO, 2003) ${ }^{5}$, esto no impide que, desde hace unos años, a medida que la inflación de diplomas se insinúa, se asista a la consolidación y generalización progresiva de nuevos espacios estratégicos. La generosidad estructural de los estudios, algo que en el fondo fue sólo garantizado por la escasez relativa de los diplomas, incita a la búsqueda de nuevas prácticas de reproducción social. Por supuesto, el punto es controvertido puesto que en mucho dependerá de la capacidad de la economía a producir empleos calificados en los años que

5- Algo que está lejos de ser insignificante. Como lo muestra el estudio de Núñez (2004a), es entre los sectores populares que la productividad de los estudios universitarios es la más alta (dado sus orígenes sociales el incremento de ingresos es más significativo con respecto a sus familias que entre los sectores medios altos). 
vienen, pero, indudablemente, Chile vive el fin de un modelo malthusiano de titulación en donde la rareza de los diplomas garantizaba a sus propietarios una rentabilidad resguardada. La conciencia de haber ingresado en una nueva fase está muy presente, por ejemplo, entre los profesionales cuando evocan la intensificación de la competencia laboral:

La competencia ha crecido enormemente. $\mathrm{Si}$ tú piensas que cuando yo egresé en Chile egresaban 70 psicólogos al año, y hoy salen $1.300 \ldots$... 0 sea en veinte años esta cuestión ha cambiado mucho en términos de mercado.

Al punto que, a diferencia de los sectores populares y las bajas capas medias, cuyas estrategias de estudio están confinadas a las credenciales nacionales, los hijos de la élite y de las capas medias altas incluyen cada vez más en sus estrategias de estudios, ya no sólo a las universidades nacionales más prestigiosas, sino también a estudios de posgrado en el extranjero.

\section{La formación continua}

La fuerte expansión de masters y doctorados, o de cursos de capacitación a todo nivel, es también una dimensión importante de la valoración de los estudios en Chile. Sin embargo, aquí también el contraste es fuerte entre los grupos sociales. Entre las clases medias, en la totalidad de los casos en que esta experiencia fue evocada, el objetivo explícito fue obtener una mejora profesional o salarial. Esta mujer ingeniera lo resume claramente: para tener un ascenso, nos dice, hay que estudiar porque "el dinero viene con el cargo". Una afirmación que para muchos es tanto más evidente que tarde o temprano para escalar posiciones en una firma aparece un techo, y que para pasar al otro lado hay que tener mayor titulación. Por supuesto, tarde o temprano esta lógica descubre una ecuación estructural: la progresión de los títulos supera al número de plazas de promoción ofertadas. Problema clásico de la adecuación entre la formación y el empleo que, sin ser aun general en Chile, conoce ya algunas modalidades patentes de saturación en ciertos sectores o empresas. En una situación de este tipo, inevitablemente aparece un sentimiento de malestar cuando, a pesar de la obtención del título, no se da la promoción, y sobre todo cuando ésta es interpretada como habiendo sido tomada desde criterios que no corresponden a los que se esperaban (ESPINOZA; SOTO, 2008).

Entre los sectores populares, la formación continua, sin que esto suponga una ausencia radical de toda dimensión estratégica, es evocada de otra manera. En el fondo, lo que se valora es el poder seguir aprendiendo, y en el marco de esta valorización general de los estudios, lo importante es aprender lo que se puede. Que el lector no minimice la fuerza de voluntad, tan frecuente entre los miembros de este grupo social, vinculada al capacitarse. Una fuerza como la de una mujer de sectores populares que, entrevistada en el salón de su domicilio, nos habló de la importancia de los estudios, de los estudios secundarios de sus hijos pero también de sus propios estudios de capacitación, enunciándolo desde una pieza en la cual los muros estaban llenos de los diplomas obtenidos por cada uno de los miembros de la familia. 0 sea, en los sectores populares la capacitación es siempre un acto de esfuerzo; a veces, pero solamente a veces, un recurso estratégico hábilmente controlado. El acto - el proyecto - de estudiar prima así muchas veces sobre otros tipos de cálculo. "Estudié hasta octavo", nos contó una mujer que trabajaba como cocinera, pero dado que se siente "inteligente para aprender", ahora está "estudiando alimentación” pero ya estudió computación, “también plomería, todo, porque han salido cursos para especializarse, yo me arriesgo a todo porque soy buena para aprender".

\section{Sectores sociales y generaciones}

Retengamos una conclusión mayor de este recorrido: a pesar de acentuaciones muy 
diferentes según el nivel de estudio, la confianza en el mérito escolar, y en el horizonte de justicia que éste traza para la escuela, es particularmente fuerte hoy en día en Chile. Incluso cuando se viene "de abajo", como dice una mujer autoempresaria de origen popular, es posible gracias a "una buena formación lograr lo que uno quiere". Esta confianza en la rentabilidad de los estudios empieza a cuestionarse o a erosionarse a medida que el incremento de los diplomas, y la transformación de las reglas de la competencia, se expanden. Pero aun así, la confianza en el valor de los estudios sigue siendo real entre muchos de los individuos de sectores populares entrevistados. Perteneciendo a una generación que no tuvo acceso a los estudios superiores o terciarios, muchos de ellos ven en el pago de los estudios de sus hijos la mejor garantía para su futuro. A sus ojos, basta con tener buenas notas en la universidad, y lograr pagar una universidad, para que las puertas del éxito (esas que siempre estuvieron cerradas para ellos) se abran para sus hijos. Los testimonios son conmovedores en lo que indican de amor parental y de anhelo de mejora social. Esforzándose en pagar los estudios superiores de su hija, un plomero expresa una verdadera preocupación por comprar los libros que ella necesita,

[...] yo no conocía el libro, pero ellos lo conseguian, 'toma aquí está la plata'. Estudia, no te preocupes, estúdiame no más, tráeme buenas notas, si me traes buenas notas yo te pago todos los estudios, pero sin no me traes y me traes malas notas no te voy a ayudar y vas a tener que trabajar.

La toma de conciencia es una evidencia compartida. Comenta un obrero:

A ver, la mejor herencia que uno le deja a los hijos es el estudio porque teniendo estudios se abren más posibilidades, porque fue duro para mí porque yo no tengo la enseñanza media completa... Yo postulé, por ejemplo, a una máquina nueva que hicieron acá en la empresa, tenía todas las condiciones para estar ahí, los conocimientos, la experiencia, lo único que falló es que no tenía la enseñanza media completa.

A sus ojos, como a los ojos de tantos otros padres de sectores populares, si su hijo hace estudios superiores, tendrá, sin lugar a dudas, mayores chances que las que él tuvo.

La confianza en el valor de los estudios entre los sectores populares presenta ya una fisura generacional. Entre los entrevistados más viejos (mayores de 45 años) el relato asocia estrechamente, al evocar la propia trayectoria escolar, dos elementos. Por un lado, la idea de la relativa rareza de esta experiencia, el hecho que para lograrla se requería de un entorno familiar particular, y, sobre todo, de aptitudes excepcionales y rápidamente reconocidas por el sistema educativo. Era solamente dentro de estas coordenadas que la escuela se convertía en un recurso posible de movilidad para algunos de ellos. Por otro lado, esta experiencia prolongada de estudios se dio en una sociedad en la cual los diplomas, a causa de su carácter malthusiano, garantizaban a aquellos que (entre los sectores populares) lograban obtenerlos, una integración efectiva en las capas medias. 0 sea, entre los mayores de nuestra muestra, el valor del diploma era indisociable a la vez de una singularidad (ser un buen alumno) y de un momento (su obtención aseguraba el ingreso en las capas medias). Desde esta óptica, para todos ellos, sus hijos se encuentran en un proceso de movilidad escolar ascendente.

Entre los más jóvenes de nuestra muestra (menores de 35 años), la experiencia es diferente y más ambigua. Los dos postulados anteriores se han modificado. Por un lado, en efecto, los estudios superiores forman parte cada vez más del horizonte de posibles de los sectores populares: en el año 2002, 44\% de padres con formación media tenían hijos con educación superior (TIRONI, 2005). Una aspiración que la expansión de un verdadero mercado 
universitario a nivel nacional, con calidad y precios muy disímiles, acompaña y sostiene (MONCKEBERG, 2007). Por otro lado, incluso si por el momento de manera un tanto difusa, se empieza a imponer la idea que la obtención de los diplomas es insuficiente, que el valor de éstos se degrada a medida que se expande la certificación escolar, y que lo que hace la diferencia por ende no es más el diploma en sí mismo, sino su lugar de obtención y, como lo veremos, la red que lo acompaña. La diferencia se jugaría a otro nivel: además de los diplomas, "hay que tener muchos contactos". Para las nuevas generaciones si los padres son, pues, un límite escolar a superar (DÁVILA; GHIARDO; MEDRANO, 2005) ${ }^{6}$, progresivamente este objetivo se inserta en el desajuste que prevén entre sus aspiraciones socio-económicas y sus posibilidades para cumplirlas (NÚÑEZ; MIRANDA, 2009).

El contraste es a veces mayúsculo entre la visión de los viejos y la de los jóvenes. Entre los últimos, como bien lo resume Bengoa (2009), se expresa una doble percepción: el hecho de ser más que sus padres y al mismo tiempo de verse menospreciados por los antiguos ricos. 0 sea, ahí donde los padres de los sectores populares ven sistemáticamente a sus hijos en posición de movilidad educativa ascendente, los hijos tienen una percepción distinta, sin duda más justa, puesto que miden con más precisión el diferencial de valor de los diplomas. Saben que el reconocimiento del mérito no se obtiene solamente con un diploma; su reconocimiento es muchas veces dependiente de una red. Aquí está el meollo de la cuestión.

\section{Las redes o el atajo hacia el éxito}

Chile es hoy, como tantas otras sociedades nacionales, una mezcla de clase y de meritocracia, o sea, de herencia y de mérito. El valor del mérito se activa, en todo

6- Según la Encuesta nacional de la juventud del año 2008, hasta un $29,5 \%$ de los estudiantes secundarios piensa que con un título de enseñanza media "no se puede aspirar a nada" (INJ, 2009: 74) caso, en un país que se caracteriza por altas tasas de movilidad social en un contexto de una desigualdad acentuada y mantenida por mecanismos de cierre formales e informales (TORCHE, 2005). Sin embargo, a pesar de los obstáculos, muchos individuos piensan que la sociedad chilena está más orientada al logro individual y a la meritocracia que lo que objetivamente revelan las trayectorias (NÚÑEZ, 2004b). En consonancia con esto, la fuerza de la expansión del mérito en Chile hoy, se expresa tanto por testimonios de su realización como por el vigor de las denuncias de su cristalización.

Los obstáculos al reconocimiento del mérito producen un fuerte sentimiento de injusticia y frustración. Si la lista de obstáculos al mérito es muy diversa (discriminaciones de género o étnicos-, lugares de trabajo bajo la influencia de juegos de poder, insuficientes alicientes en el trabajo), uno de ellos es particularmente significativo -las redes- porque cuestiona, directamente, el valor meritocrático de la escuela.

En Chile, las redes son estrategias que suponen formas particulares de contrato entre parientes o amigos que implican intercambios de favores y de amistad. Dan, así, lugar a un trato preferencial o a una ayuda, sobre todo, cuando hacen frente a una dificultad para acceder a un bien o servicio, o cuando tienen una necesidad económica. Este sistema reposa, pues, sobre reglas de endeudamiento y de obligatoriedad recíprocos en el marco de una ideología de la amistad basada en una lógica de favores y devolución de favores. Para muchos entrevistados es necesario disponer de un conjunto de relaciones sociales (de compadrazgo, de clientelismo, de padrinazgo, de amistad) porque éstas juegan un papel decisivo en la reproducción de una posición social. Y si todos recurren a las redes o los contactos, sin duda una forma de capital social (BOURDIEU, 1980), las redes tienen características muy distintas según los grupos sociales. Limitémonos a evocar tres de entre ellas: 


\section{Las redes y los grupos sociales}

En primer lugar, el recurso a las redes está particularmente presente en los sectores más acomodados. Pero, y esto es central, una diferencia es notoria a nivel de las capacidades para la movilización de las redes e incluso a nivel de una toma de posición crítica respecto a ellas, entre aquellos que pertenecen a lo que se podría llamar una clase media en ascenso, y aquellos cuya pertenencia a este grupo social es más estructural y de vieja data, la clase media instalada. Los primeros con menos redes, pero con un rápido y constante aprendizaje de las estrategias para su movilización, desarrollan críticas con mayor amargura que los segundos. En todo caso, el uso de una red fue admitido por tres cuartos de los hombres y la mitad de las mujeres entrevistadas de las capas medias contra apenas un cuarto de los miembros, hombres y mujeres, de los sectores populares. Por supuesto, esto puede ser interpretado como resultado de los diferenciales de capital social en ambos grupos ${ }^{7}$.

En segundo lugar, si la distinción entre lazos fuertes y débiles, se ha convertido desde los trabajos de Granovetter (1973) en una diferencia clásica en lo que concierne a la relación entre tipos de redes y obtención de empleos, en los testimonios recaudados, y sin que esta distinción pierda del todo su valor, es otro aspecto el que fue traído a la luz. En nuestras entrevistas, la distinción entre lazos débiles que sirven para hacer contactos (tener acceso a la buena información) y lazos fuertes (aquellos que en el sentido más preciso del término se pueden concebir como una red) se desdibujan una y otra vez. 0 mejor dicho, los lazos débiles (los contactos) tienen de una $\mathrm{u}$ otra manera que estar insertos dentro de lazos fuertes (una red) para obtener su verdadero potencial práctico. De allí que, más allá de la mayor frecuencia con la cual los miembros de las clases medias evocan las redes con respecto

7- Lechner señala que "mientras que un $56 \%$ del grupo socioeconómico alto posee capital social, sólo un 27\% del estrato bajo dispone de él” (2006, p. 561). a los sectores populares, ambos las evocan desde un saber similar: el contacto sólo tiene sentido práctico duradero inserto en una red (ESPINOZA, 1995).

En tercer lugar, y a pesar de lo anterior, la distinción es clara entre los sectores populares, quienes en sus relatos dan cuenta más bien de pitutos (contactos) que de verdaderas redes, y las clases medias, que evocan tanto el contacto como el apoyo más estructural y permanente de auténticas redes. Donde las capas medias usan las redes para mejorar su acceso a las instituciones formales (BAROZET, 2006), en los sectores populares, los contactos, incluso cuando toman la forma de redes, tienden a operar como un mecanismo informal de protección frente a la exclusión (y muchas veces frente a los límites de las instituciones). Un proceso de acceso a privilegios que tiende incluso a transmutarse en el caso de las élites en una auténtica argolla (CONTARD0, 2008).

\section{¿Otra versión del mérito?}

Vayamos a lo esencial. Para los individuos entrevistados, y cualquiera que sea la forma o la densidad de una red, lo importante es saber tejerla y sostenerla. Es esta verdad la que, como lo iremos viendo, complejiza profundamente la relación que los individuos tienen con el mérito. $\mathrm{Si}$ es cierto que las redes por sus diferenciales introducen una cuña en el mérito, sin embargo, como los mismos individuos lo afırman, incluso cuando se dispone de redes es imprescindible saber activarlas, sostenerlas, trabajarlas, lo que, curiosamente, engendra una forma específica sino necesariamente de mérito por lo menos de esfuerzo. Cierto, muchas redes se heredan, pero incluso éstas deben ser sostenidas, y en último análisis, la responsabilidad de su mantenimiento, dada la trama social en la que se halla preso el individuo, recae sobre él. Lo importante es, pues, saber usar las redes. En verdad, las redes se tienen y se trabajan. 0 mejor dicho, las redes se tienen porque se trabajan. 
Las redes son un dato de la realidad que aparece espontáneamente en los testimonios como un elemento de distorsión social que muchos individuos dicen, en principio, rechazar. Un rechazo que podría denominarse moral y formal en aquellos que pertenecen a las clases medias establecidas; un rechazo amargo y vivencial en aquellos cuya pertenencia a este sector es más bien reciente, o incluso entre los sectores populares en ascensión, y que, contando con las herramientas educativas y los recursos económicos son conscientes de su precariedad comparativa en términos de redes. Sin embargo, en un segundo momento, y no obstante lo anterior, muchas de estas mismas personas pasaron de la denuncia de las redes hacia el reconocimiento, incluso a regañadientes, de la habilidad de aquellos que sabían mantener los contactos.

Al lado de los diplomas, por encima de ellos, se impone esta otra lógica de reproducción social. Si no se es miembro de una red, las puertas se cierran. Si se es miembro de la red indicada, las cosas se dan naturalmente. Un empresario señala, así, cómo a su padre, a pesar de su edad, "no le costó mucho reubicarse (gracias a sus redes)", a tal punto para él, sin menoscabo de las competencias técnicas que se deben poseer, se impone "el tema éste de la amistocracia, además del tema técnico, sí, te tienen que recomendar".

La fuerza de esta imagen se reduplica por el tipo de representación que se tiene de las mismas. No sólo hay una enorme fe en la eficacia de las redes sino que la percepción de las mismas se asocia con una imagen de una sociedad construida en torno a círculos cerrados, lo que aumentaría la condensación de poder. Por supuesto, si la percepción de argollas absolutamente cerradas es tal vez excesiva, esto no impide que muchas redes se caractericen por su fuerte tendencia a la clausura. Nada lo ilustra mejor que las redes que se mantienen desde la experiencia escolar. La clausura está de facto impuesta por la experiencia pasada: en ese grupo reducido de amigos del colegio de infancia, nadie puede añadirse a la lista.

\section{La contaminación: el colegio como red}

En toda sociedad, en todo período histórico, el recurso a las redes y a los contactos personales, el a veces denominado capital social, es un recurso importante en las estrategias de movilidad tanto individuales como colectivas. Pero en Chile este recurso consuetudinario es objeto hoy de críticas severas y en muchos casos inéditas. Detrás de estas críticas no es, por lo tanto, una simple continuidad lo que se vislumbra, sino una nueva demanda de justicia. Dicho en otros términos, que las redes puedan ser consideradas desde cierta perspectiva como un recurso positivo y deseable no anula el hecho que ciertas funciones de las mismas puedan ser evaluadas negativamente (lo que transforme su valor y legitimidad).

Repitámoslo: la utilización de las relaciones sociales como recursos (o capital) es un hecho generalizado en todas las sociedades. En este sentido, no se debe concebir, por ejemplo, el compadrazgo, y aún más el clientelismo, como elementos idiosincráticos propios de la región (puesto que estos recursos interactivos también están presentes en otras sociedades desde el África subsahariana hasta los Estados Unidos). Pero su presencia es disímil y es esta disimilitud lo que es decisivo.

La reproducción y la transmisión de una posición social es por doquier una de las grandes preocupaciones de la familia. En América Latina esta realidad, siempre presente, pudo durante años efectuarse en el marco de una aparente despreocupación, a tal punto las distancias sociales se percibían como insalvables, a tal punto, incluso en caso de accidente mayor, el rebusque de clase se vivía como un antídoto eficaz. Es esta realidad, que se ha modificado en los últimos lustros a medida que la evidencia de la inconsistencia posicional se ha generalizado (ARAUJO; MARTUCCELLI, 2011) y con ella la necesidad para las familias de hacer un uso plenamente consciente y estratégico de la herramienta escolar. 
Esquematicemos el razonamiento: si la universidad o los diplomas terciarios son un nivel discriminante en el ámbito laboral, las redes que se tejen durante la escolaridad primaria y sobre todo secundaria consolidan una barrera más o menos infranqueable. Por supuesto, la universidad es también un lugar importante en el cual se establecen contactos que serán muchas veces decisivos a lo largo de toda la vida laboral. Una evidencia que muchos relatos enuncian claramente, ya sea para juzgar positiva o negativamente su centro de estudios - lo importante termina siendo menos la calidad educativa que se recibió que la consistencia de las redes que se tejieron en ellas. Sin embargo, y sin negar lo anterior, en las estrategias familiares de las clases medias una importancia distintiva se otorga a la escuela primaria y secundaria. La razón es simple: es alrededor de estos niveles educativos que se traza la verdadera frontera entre grupos sociales. En todo caso, en nuestra investigación, como lo han mostrado otros estudios también (RACZYNSKI, 2010), la desproporción fue enorme entre el interés $\mathrm{y}$, las competencias disimiles que los padres expresaron en lo que concierne a la elección del colegio de los hijos según su origen social.

La dimensión estratégica no está por supuesto del todo ausente entre los sectores populares. Una empleada doméstica nos contó así, por ejemplo, que escogió el centro escolar de sus hijos por "la reputación del colegio, todos me han dicho que es bueno. Es un colegio católico y es bueno... Desde los cuatro años están dando examen los niños para entrar al colegio". "Bueno, mis hijos son lo más importante en mi vida, pretendo darles lo mejor, pretendo que estudien, los tengo en buenos colegios", nos asegura un empleado, sin lograr, empero, darnos demasiadas precisiones sobre la calidad de éstos. Pero, al lado de testimonios de esta índole, y que ilustran un cierto nivel de conciencia, en otros, manifiestamente, se trata de una preocupación totalmente ausente: algunos no lograron recordar por ejemplo del nombre del colegio donde van sus hijos, y otros, como un mecánico, resumió la elección del colegio de su hijo de manera simple: "elegimos el que queda más cerca de la casa”.

El contraste es abismal con respecto a las actitudes, preocupaciones, estrategias y negociaciones presentes entre las clases medias. Obviamente, esto no quiere decir, que estos grupos se ocupen mejor de sus hijos. Lo que sí indica es que las familias usan de manera distintas la escolaridad como vía de reproducción social. Los testimonios en las clases medias fueron apabullantes a la hora de indicar la clara conciencia que se trataba de un momento álgido de decisión familiar. Desde la elección del lugar de residencia hasta la orientación valórica del colegio (especialmente en los sectores más acomodados) (THUMALA, 2007) pasando por el ranking académico, todos estos factores son parte de lo que se evalúa en las negociaciones entre los padres de cara al importante esfuerzo familiar que supone asumir durante un lapso muy extendido de tiempo costosas pensiones escolares, que, en porcentaje de ingresos familiares, están entre las más altas del mundo (MAYOL, 2012). Las razones de la elección difieren fuertemente entre sí. Pero el objetivo es siempre similar: se trata de obtener credenciales a la vez diferencialmente rentables en el mercado de trabajo y marcadores de una posición social.

La escuela no es una red más. No es nunca, en todo caso, una red cualquiera. Dado el costo que implica tejerla, tanto en términos temporales como por supuesto económicos, esta modalidad de capital social forma parte de una las garantías más tenaces de las que los individuos se sienten propietarios durante el resto de sus vidas. No es por ello raro que los comentarios evoquen la importancia de tener una buena una red. Una dimensión particularmente activa entre las élites (PNUD, 2004). Uno de los entrevistados, hablando de su colegio, comentó así que "hasta el día de hoy me invitan, yo he ido a algunas cosas, hay un registro, la iglesia conservadora trabaja muy 
bien y tú eres de acá para siempre, eres de la familia y te lo hacen sentir", y eso que él se apartó, religiosa y políticamente de ese sector social. Para otros, la importancia de este tipo de red sería tal que no dudan en hacer de su ausencia la clave de sus dificultades presentes. No haber ido a un buen colegio sería un obstáculo insalvable.

Para los miembros de las clases medias establecidas, la carrera, la verdadera, todavía se empieza y se gana ahí -en el colegio secundario. Y no solo, como lo piensan tantos padres de sectores populares, e incluso de las clases medias ascendentes, más tarde y en la Universidad. Un ejecutivo, sin cinismo, lo explica hablando del colegio al que van sus hijos:

[...] un colegio así no es fácil de financiar, además que un colegio así te da herramientas para el futuro, desde un punto de vista laboral y de contactos [...] Yo estudié construcción civil en la Universidad Católica, un compañero mío que estudió en el liceo y que estudió en la Católica probablemente desde el punto de vista académico después de egresado de la universidad sea igual o mejor que yo, pero lamentablemente por un tema que pasa a ser ya de... de donde viene uno, no tienen la misma suerte que ese que se formó en un colegio en donde hay contactos, amigos. Influye mucho más el colegio donde uno se formó que la misma universidad.

Esta estrategia revela una verdadera contaminación entre los dos caminos del éxito en Chile. Si para los sectores populares, las redes se oponen al reconocimiento del mérito por certificación escolar; para las clases medias, las certificaciones y los centros de estudio son una red. A la separación de los caminos en el primer caso, se le contrapone la superposición de los mismos en el segundo. Para ganar en el juego del mérito no basta con tener más credenciales educativas que otros. Es imperioso tener las verdaderas buenas credenciales y poder tejer las cosas como se debe. Un juego en donde prima una evidente asimetría de información.

\section{Conclusión}

Si la sociedad chilena es globalmente percibida como siendo más competitiva hoy que ayer, y por ende más abierta al reconocimiento del mérito, esta innegable percepción de mejoría se traduce por un incremento de un sentimiento de injusticia, el que alimenta una explosión de frustraciones. Para todas las personas entrevistadas, hay dos y solo dos grandes estrategias para la movilidad social: o se poseen redes o se han obtenido credenciales escolares. Salvo que, como lo hemos visto, ahí donde las clases medias tienden a asociar estrechamente ambos recursos (a término los diplomas son la base de una red), entre los sectores populares, los estudios son concebidos como una manera de paliar su ausencia inicial de redes. Entre los sectores populares lo que prima es, pues, una confianza paradójica en el mercado escolar: por un lado, confían en él y en sus promesas de movilidad social (en todo caso, no entrevén otra vía posible de ascensión), pero, por el otro lado, se enfrentan con la experiencia cotidiana de los límites, desde el costo de las matrículas escolares hasta la toma de conciencia progresiva de las disparidades de valor existentes entre los diplomas.

La fuerza de la cuestión del mérito en su doble filiación, tanto desde el modelo neoliberal como desde un más antiguo anhelo de igualdad y justicia, da cuenta de la centralidad de los debates sobre la escuela en Chile desde las manifestaciones de los estudiantes secundarios en el 2006, de los universitarios desde el 2011 y sobre todo en la agenda política del segundo gobierno de Bachelet (2014-18). Alrededor del reconocimiento del mérito escolar se asocian estrechamente cuestiones de justicia social, de eficacia económica y de expectativas familiares e individuales. 


\section{Referencias}

ARAUJO, Kathya; MARTUCCELLI, Danilo. Desafíos comunes. Santiago: LOM, 2012.

ARAUJO, Kathya; MARTUCCELLI, Danilo. La inconsistencia posicional: un nuevo concepto sobre la estratificación social. Revista CEPAL, Santiago, n. 103, p. 165-178, abr. 2011.

BAROZET, Emmanuelle. El valor histórico del pituto: clase media, integración y diferenciación social en Chile. Revista de Sociología, Santiago, n. 20, p. 69-96, 2006.

BENGOA, José. La comunidad fragmentada. Santiago: Catalonia, 2009.

BOURDIEU, Pierre. Le sens pratique. Paris: Minuit, 1980.

BRAVO, David. Trabajo: dignidad y cambios. El mercado laboral chileno. In: Tironi Barrios, Eugeni et al. Cuánto y cómo cambiamos Ios chilenos: balance de una década. Santiago: INE, 2003. p. 191-249.

BRUNNER, José Joaquín et al. Calidad de la educación: claves para el debate. Santiago: Universidad Adolfo Ibáñez y RIL, 2006.

BRUNNER, José Joaquín. La educación en la percepción de la gente. Encuesta Nacional UDP, Santiago, p.53-62, 2007.

CEPPE - Centro de Estudios de Políticas y Prácticas de Educación. Notas para educación. Santiago: CEPPE, 2011. Nota n. 9, nov. 2011.

CIDE - Centro de Investigación y Desarrollo de la Educación; ENCUESTA A ACTORES DEL SISTEMA EDUCATIVO, 9., Santiago, 2012. Encuesta...: CIDE, 2012.

CHILE. Ministerio de Educación. Chile en el panorama educacional internacional OCDE: avances y desafíos. Evidencias, Santiago, v. 2, n. 18, p. 1-8, 2013a.

CHILE. Ministerio de Educación. Sistemas universitarios modernos: diversidad y calidad institucional. Evidencias, Santiago, v. 2, n. 16, P. $1-7,2013 b$.

CONTARDO, Oscar. Siútico. Santiago: Vergara, 2008.

DÁVILA, Oscar; GHIARDO, Felipe; MEDRANO, Carlos. Los desheredados. Valparaíso: Cidpa, 2005.

DUBET, François. Injustices. Paris: Seuil, 2006.

ENGEL, Eduardo; NAVIA, Patricio. Qué gane el más mejor. Santiago: Debate, 2006.

ESPINOZA, Gabriela; SOTO, Alvaro. El vínculo que establece el trabajador o trabajadora con la empresa flexible: el caso de una empresa de servicios. In: SOTO, Alvaro (Ed.). Flexibilidad laboral y subjetividades. Santiago: LOM, 2008. p. 279-309.

ESPINOZA, Vicente. Redes sociales y superación de la pobreza. Revista de Trabajo Social, Santiago, n. 66, p. 31-44, 1995.

GAYO, Modesto. Movilidad educacional y valoraciones individuales en Chile a principios del siglos. In: Encuesta Nacional UDP, 21., Santiago, 2007. Encuesta... Santiago: CIDE, 2007. p. 63-73.

GRANOVETTER, Mark. The Strenght of Weak Ties. American Journal of Sociology, Chicago, v. 78, n. 6, p. 1360-1380, may 1973.

INJ - Instituto Nacional de la Juventud; Encuesta nacional de la juventud, 5., Santiago, 2009. Encuesta... Santiago: FNSP, 2009. LECHNER, Norbert. Las sombras del mañana. Santiago: LOM, 2006.

LEÓN, Arturo; MARTínEZ, Javier. La estratificación social en Chile hacia fines del siglo XX. In: FRANCO, Rolando; LEÓN, Arturo; ATRICA, Raúl (Eds.). Estratificación y movilidad social en América Latina. Santiago: LOM, 2007. p. 303-337. 
MANZI, Jorge. El acceso segmentado a la educación superior en Chile. In: DÍAZ-ROMERO, Pamela (Ed.). Caminos para la inclusión en la educación superior. Santiago: Fundación Equitas, 2006. p. 187-204.

MAYOL, Alberto. El derrumbe del modelo. Santiago: LOM, 2012.

MÉNDEZ, María Luisa; GAYO, Modesto. El perfil de un debate: movilidad y meritocracia. Contribución al estudio de las sociedades latinoamericanas. In: FRANCO, Rolando; LEÓN, Arturo; ATRICA, Raúl (Eds.). Estratificación y movilidad social en América Latina. Santiago: LOM, 2007. p. 121-157.

MICHAUD, Yves. Qu'est-ce que le mérite? Paris: Bourin, 2009.

MIZALA, Alejandra; ROMAGUERA, Pilar. Remuneraciones y tasas de retorno de los profesionales chilenos. In: BRUNNER, José Joaquín; MELLER, Patricio (Eds.). Oferta y demanda de profesionales y técnicos en Chile. Santiago: RIL, 2004. p. 171-209.

MONCKEBERG, María Olivia. El negocio de las universidades en Chile. Santiago: Debate, 2007.

NúÑEZ, Javier. Ni Gonzáles ni Tapia: clasismo versus meritocracia en Chile. Santiago: Universidad de Chile / Departamento de la Economía, 2004a.

NÚÑEZ, Javier. Discriminación y meritocracia en el mercado laboral en Chile. Economía y Administración, Santiago, n. 147, p. $4-8,2004 b$.

NÚÑEZ, Javier; MIRANDA, Leslie. La movilidad intergeneracional del ingreso y la educación en Chile. In: JOIGNANT, Alfredo; GUELL, Pedro (Eds.). El arte de clasificar a los chilenos. Santiago: UDP, 2009. p. 83-101.

PNUD. El poder ¿para qué y para quién? Santiago: PNUD, 2004. Informe del desarrollo humano en Chile.

RACZYNSKI, Dagmar. Hacia una estrategia de validación de la educación pública-municipal: valoraciones y demandas de las familias. Santiago: Mineduc, 2010.

RIVAS, Ricardo. Logro educacional en jóvenes y clase social de origen: el caso del gran concepción. Última Década, Valparaíso, n. 28, p. 123-142, 2008.

SAPELLI, Claudio. Los retornos a la educación en Chile: estimaciones por corte transversal y por cohortes. Santiago: Instituto de Economía, Pontificia Universidad Católica de Chile, 2009. Documento de trabajo n. 349.

STABILI, María Rosa. El sentimiento aristocrático. Santiago: Andrés Bello: Centro de Investigaciones Diego Barros Arana, 2003.

THUMALA, María Angélica. Riqueza y piedad. Santiago: Debate, 2007.

TIRONI, Eugenio. El sueño chileno. Santiago de Chile: Taurus, 2005.

TORCHE, Florencia. Unequal but fluid social mobility in Chile in a comparative perspective. American Sociological Review, Nashville, v. 70, n. 3, p. 422-450, 2005.

Recebido en: 14.10.2014

Aprobado en: 11.03.2015

Kathya Araujo es profesora en la Universidad Academia Humanismo Cristiano, Chile.

Danilo Martuccelli es profesor de sociología en la Faculdad de Ciencias Humanas y Sociales de la Universidad ParisDescartes, Université Sorbonne Paris Cité. Miembro senior del Institut Universitaire de France, Centre de Recherches sur Liens Sociaux (CERLIS). 